\title{
EXISTENCE AND BEHAVIOR OF SOLUTIONS FOR A WEAKLY COUPLED SYSTEM OF REACTION-DIFFUSION EQUATIONS
}

\section{Kiyoshi Mochizuki and Qing Huang}

ABSTRACT. We consider the weakly coupled system of reaction-diffusion equations

$$
u_{t}=\Delta u+|x|^{\sigma_{1}} v^{p}, \quad v_{t}=\Delta v+|x|^{\sigma_{2}} u^{q},
$$

where $x \in \mathbf{R}^{N}(N \geq 1), t>0, p, q \geq 1$ with $p q>1$ and $0 \leq \sigma_{1}<N(p-1)$, $0 \leq \sigma_{2}<N(q-1)$. Put

$$
\alpha=\frac{2(p+1)}{p q-1}, \quad \beta=\frac{2(q+1)}{p q-1}, \quad \delta_{1}=\frac{\sigma_{2} p+\sigma_{1}}{p q-1}, \quad \delta_{2}=\frac{\sigma_{1} q+\sigma_{2}}{p q-1},
$$

and let $I^{a}$ and $I_{a}(a \geq 0)$ be the spaces of nonnegative, bounded continuous functions satisfying

$$
\limsup _{|x| \rightarrow \infty}|x|^{a} \xi(x)<\infty \quad \text { and } \quad \liminf _{|x| \rightarrow \infty}|x|^{a} \xi(x)>0,
$$

respectively. At $t=0$, initial values $\left(u_{0}, v_{0}\right) \in I^{\delta_{1}} \times I^{\delta_{2}}$ are prescribed. It is proved that if $\max \left\{\alpha+\delta_{1}, \beta+\delta_{2}\right\} \geq N$ or if $u_{0} \in I_{a}$ with $a<\alpha+\delta_{1}$ or $v_{0} \in I_{b}$ with $b<\beta+\delta_{2}$, then every nontrivial nonnegative solution is not global in time; whereas if $\max \left\{\alpha+\delta_{1}, \beta+\delta_{2}\right\}<N$ and $\left(u_{0}, v_{0}\right) \in I^{a} \times I^{b}$ with $a>\alpha+\delta_{1}, b>\beta+\delta_{2}$, then there exist both global solutions and nonglobal solutions. Moreover, we obtain the asymptotic behavior as $t \rightarrow \infty$ of the global solutions.

\section{Introduction}

We consider nonnegative solutions of the initial value problem for a weakly coupled system

$$
\begin{array}{ll}
u_{t}=\Delta u+|x|^{\sigma_{1}} v^{p}, & x \in \mathbf{R}^{N}, t>0, \\
v_{t}=\Delta v+|x|^{\sigma_{2}} u^{q}, & x \in \mathbf{R}^{N}, t>0, \\
u(x, 0)=u_{0}(x), & x \in \mathbf{R}^{N}, \\
v(x, 0)=v_{0}(x), & x \in \mathbf{R}^{N},
\end{array}
$$

where $N \geq 1, p, q \geq 1$ with $p q>1$, and $0 \leq \sigma_{1}<N(p-1), 0 \leq \sigma_{2}<N(q-1)$ (if $p=1$ or $q=1$, we choose $\sigma_{1}=0$ or $\left.\sigma_{2}=0\right)$. The problem provides a simple example of a reaction-diffusion system. As a model of heat propagation in a two-component combustible mixture, $u, v$ represent the temperatures of the interacting components.

For given initial values $\left(u_{0}, v_{0}\right)$, let $T^{*}=T^{*}\left(u_{0}, v_{0}\right)$ be the maximal existence time of solutions. If $T^{*}=\infty$, the solutions are global. The global existence and nonexistence of solutions are studied by Escobedo and Herrero [2] in the case $\sigma_{1}=\sigma_{2}=0$. They

Received March 10, 1997, revised October 15, 1997.

1991 Mathematics Subject Classification: 35K57, 35B05.

Key words and phrases: blow up, large time behavior, reaction-diffusion system. 
consider nonnegative, bounded solutions, and among other things, the following results are proved there.

(I) If $2 \max \{p+1, q+1\} \geq N(p q-1)$, then $T^{*}<\infty$ for every nontrivial solution $(u(t), v(t))$ of $(1)$

(II) if $2 \max \{p+1, q+1\}<N(p q-1)$, then there exist both non-global solutions and non-trivial global solutions of (1).

In this paper, we shall extend these results to the case $0 \leq \sigma_{1}<N(p-1)$ and $0 \leq \sigma_{2}<N(q-1)$. Moreover, we shall study the behavior as $t \rightarrow \infty$ of the global solutions.

Throughout the rest of this paper, we shall use the following notations. We put

$$
\alpha=\frac{2(p+1)}{p q-1}, \quad \beta=\frac{2(q+1)}{p q-1}, \quad \delta_{1}=\frac{\sigma_{2} p+\sigma_{1}}{p q-1}, \quad \delta_{2}=\frac{\sigma_{1} q+\sigma_{2}}{p q-1} .
$$

We set $B C$ to be the space of all bounded continuous functions in $\mathbf{R}^{N}$ and for $a \geq 0$,

$$
\begin{gathered}
I^{a}=\left\{\xi \in B C \mid \xi(x) \geq 0 \text { and } \limsup _{|x| \rightarrow \infty}|x|^{a} \xi(x)<\infty\right\}, \\
I_{a}=\left\{\xi \in B C \mid \xi(x) \geq 0 \text { and } \liminf _{|x| \rightarrow \infty}|x|^{a} \xi(x)>0\right\} .
\end{gathered}
$$

Let $L_{a}^{\infty}$ be the Banach space of $L^{\infty}$-functions in $\mathbf{R}^{N}$ such that

$$
\|\xi\|_{\infty, a} \equiv \sup _{x \in \mathbf{R}^{N}}\langle x\rangle^{a}|\xi(x)|<\infty
$$

where $\langle x\rangle^{a}=\left(1+|x|^{2}\right)^{a / 2}$. Then $I^{a} \subset L_{a}^{\infty}$. The letter $C$ denotes a positive generic constant which may vary from line to line. We shall use the notation $S(t) \xi$ to represent the solution of the heat equation with initial value $\xi(x)$ :

$$
S(t) \xi(x)=(4 \pi t)^{-N / 2} \int_{\mathbf{R}^{N}} e^{-|x-y|^{2} / 4 t} \xi(y) d y .
$$

In the following, we require

$$
\left(u_{0}(x), v_{0}(x)\right) \in I^{\delta_{1}} \times I^{\delta_{2}} .
$$

As noted in $\S 2$ below, problem (1) has a unique, nonnegative solution $(u(\cdot, t), v(\cdot, t)) \in$ $I^{\delta_{1}} \times I^{\delta_{2}}$ at least locally in time.

Now the results of this paper will be summarized in the following four theorems.

Theorem 1.1. Assume $\max \left\{\alpha+\delta_{1}, \beta+\delta_{2}\right\} \geq N$. Then $T^{*}<\infty$ for every nontrivial solution $(u(t), v(t))$.

Theorem 1.2. Assume $\max \left\{\alpha+\delta_{1}, \beta+\delta_{2}\right\}<N$. Suppose also that one of the following two conditions holds

(i) $u_{0} \in I_{a}$ with $a<\alpha+\delta_{1}$ or $v_{0} \in I_{b}$ with $b<\beta+\delta_{2}$;

(ii) $u_{0}(x)$ or $v_{0}(x) \geq C e^{-\nu_{0}|x|^{2}}$ for some $\nu_{0}>0$ and some $C>0$ large enough.

Then $T^{*}<\infty$ for every solution $(u(t), v(t))$.

Under the condition $\max \left\{\alpha+\delta_{1}, \beta+\delta_{2}\right\}<N$, we have $p>1+\left(2+\sigma_{1}\right) / N$ or $q>1+\left(2+\sigma_{2}\right) / N$. In the following two theorems, we only consider the case $q>1+\left(2+\sigma_{2}\right) / N$. Similar results also are obtained when $p>1+\left(2+\sigma_{1}\right) / N$. 
Theorem 1.3. Assume $\max \left\{\alpha+\delta_{1}, \beta+\delta_{2}\right\}<N$ and $q>1+\left(2+\sigma_{2}\right) / N$. Let

$$
\left(u_{0}, v_{0}\right) \in I^{a} \times I^{b} \quad \text { with } \quad a>\alpha+\delta_{1}, \quad b>\beta+\delta_{2} .
$$

If $\left\|u_{0}\right\|_{\infty, a}+\left\|v_{0}\right\|_{\infty, b}$ is small enough, then $T^{*}=\infty$, and we have

$$
u(x, t) \leq C S(t)\langle x\rangle^{-\tilde{a}}, \quad v(x, t) \leq C S(t)\langle x\rangle^{-\tilde{b}}
$$

in $\mathbf{R}^{N} \times(0, \infty)$ where $\tilde{a} \leq a$ and $\tilde{b} \leq b$ are chosen to satisfy

$$
\alpha+\delta_{1}<\tilde{a}<\min \left\{N, N p-2-\sigma_{1}\right\}, \quad \frac{\tilde{a}+2+\sigma_{1}}{p}<\tilde{b}<\tilde{a} q-2-\sigma_{1} .
$$

Theorem 1.4. Let $(u(t), v(t))$ be the above global solution of (1).

(i) If we can choose $\tilde{a}=a[$ or $\tilde{b}=b<N]$ in (4) and if

$$
\lim _{|x| \rightarrow \infty}|x|^{a} u_{0}(x)=A>0 \quad\left[\text { or } \quad \lim _{|x| \rightarrow \infty}|x|^{b} v_{0}(x)=B>0\right],
$$

then

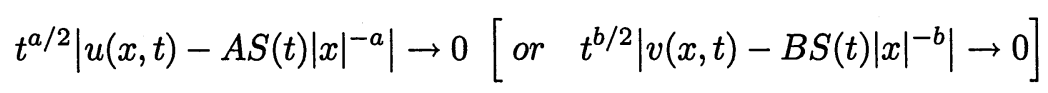

as $t \rightarrow \infty$ uniformly in $\mathbf{R}^{N}$.

(ii) If we can choose $\tilde{b}>N$ in (4), then

$$
t^{N / 2}\left|v(x, t)-M(4 \pi t)^{-N / 2} e^{-|x|^{2} / 4 t}\right| \rightarrow 0 \text { as } t \rightarrow \infty
$$

uniformly on the set $\left\{x \in \mathbf{R}^{N}|| x \mid \leq R t^{1 / 2}\right\}(R>0)$ where

$$
M=\int_{\mathbf{R}^{N}} v_{0}(x) d x+\int_{0}^{\infty} \int_{\mathbf{R}^{N}}|x|^{\sigma_{2}} u(x, t)^{q} d x d t<\infty .
$$

Remark 1.5. We put $\Delta=\{(\tilde{a}, \tilde{b}) \mid$ satisfying (5) $\}$. Then since

$$
\begin{gathered}
\min \left\{N, N p-2-\sigma_{1}\right\}-\alpha-\delta_{1}=\min \left\{N-\alpha-\delta_{1}, p\left(N-\beta-\delta_{2}\right)\right\}>0, \\
\tilde{a} q-2-\sigma_{2}-\frac{\tilde{a}+2+\sigma_{1}}{p}=\frac{(p q-1)\left(\tilde{a}-\alpha-\delta_{1}\right)}{p}>0,
\end{gathered}
$$

$\Delta$ forms a nonempty triangular domain in $\mathbf{R}_{+}^{2}$. Moreover, since

$$
\frac{\alpha+\delta_{1}+2+\sigma_{1}}{p}=\beta+\delta_{2}
$$

for any $a, b$ satisfying (3), we can choose a pair $\tilde{a} \leq a$ and $\tilde{b} \leq b$ in the domain $\Delta$. $\tilde{b}$ may be larger than $N$. In fact, we have

$$
\begin{aligned}
\min & \left\{N, N p-2-\sigma_{1}\right\} q-2-\sigma_{1} \\
& =N+\min \left\{N(q-1)-2-\sigma_{2},(p q-1)\left(N-\beta-\delta_{2}\right)\right\} \\
& >N
\end{aligned}
$$

Remark 1.6. If both $p>1+\left(2+\sigma_{1}\right) / N$ and $q>l+\left(2+\sigma_{2}\right) / N$ are satisfied and if $a, b>N$ in (3), then we have not only Theorem 4 (ii) for $v(x, t)$ but also the following result for $u(x, t)$ :

$$
t^{N / 2}\left|u(x, t)-L(4 \pi t)^{-N / 2} e^{-|x|^{2} / 4 t}\right| \rightarrow 0 \text { as } t \rightarrow \infty
$$


uniformly on the set $\left\{x \in \mathbf{R}^{N}|| x \mid \leq R t^{1 / 2}\right\}(R>0)$ where

$$
L=\int_{\mathbf{R}^{N}} u_{0}(x) d x+\int_{0}^{\infty} \int_{\mathbf{R}^{N}}|x|^{\sigma_{1}} v(x, t)^{p} d x d t<\infty .
$$

In fact, in this case, we can choose $\tilde{a} \leq a, N<\tilde{b} \leq b$ to satisfy (5) and also $N<\hat{a} \leq a$, $\hat{b} \leq b$ to satisfy

$$
\beta+\delta_{2}<\hat{b}<\min \left\{N, N q-2-\sigma_{2}\right\}, \quad \frac{\hat{b}+2+\sigma_{2}}{q}<\hat{a}<\hat{b} p-2-\sigma_{2} .
$$

Then since $I^{a} \times I^{b} \subset\left(I^{\tilde{a}} \times I^{\tilde{b}}\right) \cap\left(I^{\hat{a}} \times I^{\hat{b}}\right)$, repeating the argument of Theorem 4 (ii) yields the result.

Through these theorems, we see: (a) the pair $(p, q)$ satisfying $\max \left\{\alpha+\delta_{1}, \beta+\delta_{2}\right\}=$ $N$ gives a critical exponent of blow-up, and (b) the decay rate $(a, b)=\left(\alpha+\delta_{1}, \beta+\delta\right)$ of initial values gives another critical exponent of blow-up. The first critical exponent coincides with Fujita's classical one [3], $p=1+2 / N$ if $p=q$ and $\sigma_{1}=\sigma_{2}=0$. If $p=q$ and $\sigma_{1}=\sigma_{2}=\sigma>0$, it is reduced to the critical exponent $p=1+(2+\sigma) / N$ obtained by Bandle and Levine [1]. The critical blow-up results for these single equations were obtained later (see Hayakawa [7], Kobayashi et al. [11], Weissler [16], and Hamada [6]). Result (a) for our problem (1) is announced in Uda [15] without proof. The critical blow-up result is, however, not studied there. To show the critical blow-up, we follow the argument of Escobedo and Herrero [2]. The point is to obtain a priori estimates for global solutions. However, it seems difficult to generalize their results to our case. Therefore, we replace them by our new necessary conditions for the global existence. The second critical exponent is obtained for single equations by Lee and $\mathrm{Ni}$ [12] and for a system of equations with $\sigma_{1}=\sigma_{2}=0$ by Huang et al. [8]. Theorems 3 and 4 generalize results of [8], in which only a very special case of slow decay initial values is treated by a different method. Note that results similar to the above theorems have been obtained in Mochizuki [13] for the quasilinear equation

$$
u_{t}=\Delta u^{m}+|x|^{\sigma} u^{p}
$$

with $m>\max \{0,1-2 / N\}, p>\max \{1, m\}$, and $0 \leq \sigma<N \min \{p-1, p / m-1\}$.

The rest of the paper is organized as follows: Some preliminary results, including the existence for (1), are gathered in $\S 2$. A necessary condition for global existence is summarized in Theorem 2.5. Based on this theorem, we shall prove Theorems 1 and 2 in $\S 3$. Theorems 3 and 4 are proved in $\S \S 4$ and 5 , respectively.

\section{Preliminaries}

In order to show the local solvabilty of the Cauchy problem (1), we consider the associated integral system

$$
\begin{aligned}
& u(t)=S(t) u_{0}+\int_{0}^{t} S(t-s)|\cdot| \sigma^{\sigma_{1}}|v(s)|^{p-1} v(s) d s \\
& v(t)=S(t) v_{0}+\int_{0}^{t} S(t-s)|\cdot|^{\sigma_{2}}|u(s)|^{q-1} u(s) d s .
\end{aligned}
$$

Define

$$
\Psi(u, v)=\left(S(t) u_{0}+\Phi_{1}(v), S(t) v_{0}+\Phi_{2}(u)\right)
$$


where

$$
\begin{aligned}
\Phi_{1}(v) & =\int_{0}^{t} S(t-s)|\cdot|^{\sigma_{1}}|v(s)|^{p-1} v(s) d s \\
\Phi_{2}(u) & =\int_{0}^{t} S(t-s)|\cdot|^{\sigma_{2}}|u(s)|^{q-1} u(s) d s
\end{aligned}
$$

and for arbitrary $T>0$, consider the set

$$
E_{T}=\left\{(u, v)\left|[0, T] \rightarrow L_{\delta_{1}}^{\infty} \times L_{\delta_{2}}^{\infty}\right|\|(u, v)\|_{E_{T}}<\infty\right\}
$$

where

$$
\|(u, v)\|_{E_{T}}=\sup _{t \in[0, T]}\left\{\|u(t)\|_{\infty, \delta_{1}}+\|v(t)\|_{\infty, \delta_{2}}\right\}
$$

Then $E_{T}$ is a Banach space.

Lemma 2.1. Let $\gamma>0$ and $0 \leq \delta \leq \min \{N, \gamma\}$. Then we have for any $t>0$,

$$
\left\|S(t)\langle\cdot\rangle^{-\gamma}\right\|_{\infty, \delta} \leq \begin{cases}C(1+t)^{(-\min \{N, \gamma\}+\delta) / 2}, & \text { when } \gamma \neq N \\ C(1+t)^{(-N+\delta) / 2} \log (2+t), & \text { when } \gamma=N .\end{cases}
$$

Proof. Note that

$$
\begin{aligned}
& |x|^{\delta} S(t)\langle x\rangle^{-\gamma} \\
& =|x|^{\delta}\left\{\int_{|y|<|x| / 2}+\int_{|y|>|x| / 2}\right\}(4 \pi t)^{-N / 2} e^{-|y|^{2} / 4 t}\langle x-y\rangle^{-\gamma} d y \\
& \equiv \mathrm{I}+\mathrm{II}
\end{aligned}
$$

Here $\mathrm{I} \leq 2^{\delta} S(t)\langle x\rangle^{\delta-\gamma}$ and

$$
\begin{aligned}
\mathrm{II} & \leq|x|^{\delta} e^{-|x|^{2} / 32 t} \int_{|y|>|x| / 2}(4 \pi t)^{-N / 2} e^{-|y|^{2} / 8 t}\langle x-y\rangle^{-\gamma} d y \\
& \leq C t^{\delta / 2} S(2 t)\langle x\rangle^{-\gamma} .
\end{aligned}
$$

As is easily seen (cf., [12; Lemma 2.12]),

$$
\left\|S(t)\langle\cdot\rangle^{-\gamma}\right\|_{\infty} \leq \begin{cases}C(1+t)^{-\min \{N, \gamma\} / 2}, & \text { when } \gamma \neq N \\ C(1+t)^{-N / 2} \log (t+2), & \text { when } \gamma=N\end{cases}
$$

Thus, the desired inequality holds true.

Lemma 2.2. (i) Let $\left(u_{0}, v_{0}\right) \in I^{\delta_{1}} \times I^{\delta_{2}}$. Then $\left(S(\cdot) u_{0}, S(\cdot) v_{0}\right) \in E_{T}$ for any $T>0$, and we have

$$
\left\|\left(S(\cdot) u_{0}, S(\cdot) v_{0}\right)\right\|_{E_{T}} \leq C\left\{\left\|u_{0}\right\|_{\infty, \delta_{1}}+\left\|v_{0}\right\|_{\infty, \delta_{2}}\right\} .
$$

(ii) Let $(u, v) \in E_{T}$. Then $\left(\Phi_{1}(v), \Phi_{2}(u)\right) \in E_{T}$, and we have

$$
\left\|\left(\Phi_{1}(v), \Phi_{2}(u)\right)\right\|_{E_{T}} \leq C T\left\{\|(0, v)\|_{E_{T}}^{p}+\|(u, 0)\|_{E_{T}}^{q}\right\} .
$$

Proof. (i) is obvious from Lemma 2.1 with $\gamma=\delta=\delta_{k}(k=1,2)$.

(ii) Note that

$$
\int_{0}^{t} S(t-s)|\cdot|^{\sigma_{1}} v(s)^{p} d s \leq \int_{0}^{t} S(t-s)\langle\cdot\rangle^{\sigma_{1}-\delta_{2} p} d s \sup _{s \in[0, t]}\|v(s)\|_{\infty, \delta_{2}}^{p} .
$$


By a simple calculation, $-\sigma_{1}+\delta_{2} p=\delta_{1}<N$. Then it follows from Lemma 2.1 with $\gamma=\delta=\delta_{1}$ that

$$
\left\|S(t-s)\langle\cdot\rangle^{\sigma_{1}-\delta_{2} p}\right\|_{\infty, \delta_{1}} \leq C
$$

Thus we have

$$
\left\|\int_{0}^{t} S(t-s)|\cdot|^{\sigma_{1}} v(s)^{p} d s\right\|_{\infty, \delta_{1}} \leq C t \sup _{s \in[0, t]}\|v(s)\|_{\infty, \delta_{2}}^{p}
$$

Similarly, we have

$$
\left\|\int_{0}^{t} S(t-s)|\cdot|^{\sigma_{2}} u(s)^{q} d s\right\|_{\infty, \delta_{2}} \leq C t \sup _{s \in[0, t]}\|u(s)\|_{\infty, \delta_{1}}^{q} .
$$

These inequalities conclude the assertion (ii).

Now we can prove

Theorem 2.3. Assume that $\left(u_{0}, v_{0}\right) \in I^{\delta_{1}} \times I^{\delta_{2}}$. Then there exists $0<T \leq \infty$ and a unique pair $(u(t), v(t)) \in P_{T}=\left\{(u, v) \in E_{T} \mid u \geq 0, v \geq 0\right\}$ which solves (1) in $\mathbf{R}^{N} \times(0, T)$.

Proof. (cf. [2; Theorem 2.1]) Let $B_{R}=\left\{(u, v) \in E_{T} \mid\|(u, v)\|_{E_{T}} \leq R\right\}$. If $R$ is large enough and $T>0$ is small enough, then using Lemmas 2.2, one easily sees that $\Psi$ is a strict contraction of $B_{R} \cap P_{T}$ into itself. Thus there exists a unique fixed point which solves (1). Here we have used the fact that $P_{T}$ is a closed subset of $E_{T}$.

Next, we shall obtain a necessary condition for the global existence of solutions. Let $\rho_{\epsilon}(x)=(\epsilon / \pi)^{N / 2} e^{-\epsilon|x|^{2}}, \epsilon>0$. For a solution $(u(t), v(t)) \in E_{T}$ of (1), we put

$$
F_{\epsilon}(t)=\int_{\mathbf{R}^{N}} u(x, t) \rho_{\epsilon}(x) d x, \quad G_{\epsilon}(t)=\int_{\mathbf{R}^{N}} v(x, t) \rho_{\epsilon}(x) d x .
$$

Since $-\Delta \rho_{\epsilon}(x) \leq 2 N \epsilon \rho_{\epsilon}(x)$, the pair $\left\{2 N \epsilon, \rho_{\epsilon}(x)\right\}$ is regarded as an approximate principal eigensolution of $-\Delta$ in $\mathbf{R}^{N}$ (see e.g., Imai and Mochizuki [9]). With this fact and the Hölder inequality we easily have

$$
\begin{aligned}
& F_{\epsilon}^{\prime}(t) \geq-2 N \epsilon F_{\epsilon}(t)+C_{p} \epsilon^{-\sigma_{1} / 2} G_{\epsilon}(t)^{p}, \\
& G_{\epsilon}^{\prime}(t) \geq-2 N \epsilon G_{\epsilon}(t)+C_{q} \epsilon^{-\sigma_{2} / 2} F_{\epsilon}(t)^{q},
\end{aligned}
$$

where for $\sigma /(\tilde{p}-1)=\sigma_{1} /(p-1)$ or $\sigma /(\tilde{p}-1)=\sigma_{2} /(q-1)$,

$$
C_{\tilde{p}}=\left(\pi^{-N / 2} \int_{\mathbf{R}^{N}}|x|^{-\sigma /(\tilde{p}-1)} e^{-|x|^{2}} d x\right)^{-\tilde{p}+1} .
$$

Let us consider the system of ordinary differential equations

$$
\begin{gathered}
f^{\prime}(t)=-2 N \epsilon f(t)+C_{p} \epsilon^{-\sigma_{1} / 2} g(t)^{p} \\
g^{\prime}(t)=-2 N \epsilon g(t)+C_{q} \epsilon^{-\sigma_{2} / 2} f(t)^{q} \\
f(0)=F_{\epsilon}(0), \quad g(0)=G_{\epsilon}(0) .
\end{gathered}
$$

We say that $(f(t), g(t))$ is not global or blows up in finite time if there exists $T>0$ such that

$$
\limsup _{t \rightarrow T_{-}} \max \{f(t), g(t)\}=\infty \text {. }
$$

As an application of the standard theory of ODE, we have: 
Lemma 2.4. (i) Let

$$
Q=\left\{(f, g) \in \mathbf{R}_{+}^{2} \mid\left(C_{p}^{-1} 2 N \epsilon^{1+\sigma_{1} / 2} f\right)^{1 / p}<g<C_{q}\left(2 N \epsilon^{1+\sigma_{2} / 2}\right)^{-1} f^{q}\right\} .
$$

If $(f, g)$ solves $(13)$ on $(0, T)$ and $(f(0), g(0)) \in Q$, then $(f(t), g(t)) \in Q$ on $(0, T)$.

(ii) Let $(f(0), g(0)) \in Q$. Then $(f(t), g(t))$ blows up in a finite time.

Proof. See e.g., Qi and Levine [14; §3] or Galaktionov et al. [4, 5].

Note that there is only one equilibrium of system (13) in $\mathbf{R}_{+}^{2}$, say,

$$
P=\left(C_{1} \epsilon^{\left(\alpha+\delta_{1}\right) / 2}, C_{2} \epsilon^{\left(\beta+\delta_{2}\right) / 2}\right) \quad\left(C_{1}, C_{2}>0\right) .
$$

$P$ is a saddle point. One of the separatrices starts from 0 and runs to $\infty$. Another one intersects the $f$-and $g$-axes at $A_{\epsilon}$ and $B_{\epsilon}$, respectively. By a scaling argument, we see that

$$
A_{\epsilon}=A_{1} \epsilon^{\left(\alpha+\delta_{1}\right) / 2}, \quad B_{\epsilon}=B_{1} \epsilon^{\left(\beta+\delta_{2}\right) / 2} .
$$

Every solution $(f(t), g(t))$ of $(13)$ with the initial value $(f(0), g(0))$ lying above this separatrix runs into $Q$ and hence blows up in finite time.

As a result of these arguments and a comparison principle, we have the following:

Theorem 2.5. Let $\left(F_{\epsilon}(t), G_{\epsilon}(t)\right)$ satisfy differential inequalities (12). If

$$
F_{\epsilon}(0)>A_{1} \epsilon^{\left(\alpha+\delta_{1}\right) / 2} \quad \text { or } G_{\epsilon}(0)>B_{1} \epsilon^{\left(\beta+\delta_{2}\right) / 2}
$$

for some $\epsilon>0$, then $\left(F_{\epsilon}(t), G_{\epsilon}(t)\right)$ blows up in finite time.

\section{Proof of Theorems 1 and 2}

Throughout this section, we assume $\beta+\delta_{2} \geq \alpha+\delta_{1}$ for definiteness. We shall require the following auxiliary tools.

Lemma 3.1. Let $\left(u_{0}, v_{0}\right) \neq(0,0)$, and let $(u(t), v(t))$ be a solution of (1). Then there exist $\tau=\tau\left(u_{0}, v_{0}\right) \geq 0$ and constants $C>0, \nu>0$ such that

$$
u(\tau) \geq C e^{-\nu|x|^{2}} \quad \text { and } \quad v(\tau) \geq C e^{-\nu|x|^{2}} .
$$

Proof. Obvious (see [2; Lemma 2.4]).

Lemma 3.2. For $\sigma \geq 0$ and $\nu>0$, we have

$$
S(t)|x|^{\sigma} e^{-\nu|x|^{2}} \geq C_{\sigma}(2 t)^{\sigma / 2}(2 \nu t+1)^{-(N+\sigma) / 2} e^{-|x|^{2} / 2 t}
$$

where

$$
C_{\sigma}=(2 \pi)^{-N / 2} \int_{\mathbf{R}^{N}}|x|^{\sigma} e^{-|x|^{2}} d x
$$

Proof. We have

$$
\begin{aligned}
S(t)|x|^{\sigma} e^{-\nu|x|^{2}} & \geq(4 \pi t)^{-N / 2} e^{-|x|^{2} / 2 t} \int_{\mathbf{R}^{N}}|y|^{\sigma} e^{-(1+2 \nu t)|y|^{2} / 2 t} d y \\
& =C_{\sigma}(2 t)^{-N / 2} e^{-|x|^{2} / 2 t}\left(\frac{1+2 \nu t}{2 t}\right)^{-(N+\sigma) / 2}
\end{aligned}
$$

This proves the lemma.

Theorem 1 in the noncritical case $\beta+\delta_{2}>N$ and Theorem 2 are direct consequences of Theorem 2.5. 
Proof of Theorem 1 (noncritical case). By means of a comparison principle and Lemma 3.1, we can assume $v_{0} \in L^{1}\left(\mathbf{R}^{N}\right)$ and

$$
\int_{\mathbf{R}^{N}} v_{0}(x) d x>0
$$

The Lebesgue dominated convergence theorem then shows the existence of $\epsilon_{0}>0$ such that

$$
G_{\epsilon}(0)=(\epsilon / \pi)^{N / 2} \int_{\mathbf{R}^{N}} v_{0}(x) e^{-\epsilon|x|^{2}} d x \geq \frac{1}{2}(\epsilon / \pi)^{N / 2} \int_{\mathbf{R}^{N}} v_{0}(x) d x
$$

for any $0<\epsilon \leq \epsilon_{0}$. Since $\beta+\delta_{2}>N$, this implies that the condition of Theorem 2.5 is satisfied if $\epsilon \leq \epsilon_{0}$ is chosen sufficiently small. Thus, $\left(F_{\epsilon}(t), G_{\epsilon}(t)\right)$ blows up in a finite time.

Proof of Theorem 2. First consider the case (i). If $u_{0} \in I_{a}$ with $a<\alpha+\delta_{1}<N$, then we have

$$
F_{\epsilon}(0)=(\epsilon / \pi)^{N / 2} \int_{\mathbf{R}^{N}} u_{0}(x) e^{-\epsilon|x|^{2}} d x=\pi^{-N / 2} \int_{\mathbf{R}^{N}} u_{0}\left(\epsilon^{-1 / 2} x\right) e^{-|x|^{2}} d x .
$$

Then it follows that

$$
\epsilon^{-\left(\alpha+\delta_{1}\right) / 2} F_{\epsilon}(0) \geq C \epsilon^{-\left(\alpha+\delta_{1}-a\right) / 2} \pi^{-N / 2} \int_{\mathbf{R}^{N}}|x|^{-a} e^{-|x|^{2}} d x>A_{1}
$$

for sufficiently small $\epsilon>0$. If $v_{0} \in I_{b}$ with $b<\beta+\delta_{2}<N$, we similarly have

$$
\epsilon^{-\left(\beta+\delta_{2}\right) / 2} G_{\epsilon}(0)>B_{1}
$$

for sufficiently small $\epsilon>0$. Thus, $\left(F_{\epsilon}(t), G_{\epsilon}(t)\right)$ blows up in finite time by Theorem 2.5.

Next consider the case (ii). We then have

$$
F_{\epsilon}(0) \quad \text { or } \quad G_{\epsilon}(0) \geq C(\epsilon / \pi)^{N / 2} \int_{\mathbf{R}^{N}} e^{-\left(\epsilon+\nu_{0}\right)|x|^{2}} d x=C\left(\frac{\epsilon}{\epsilon+\nu_{0}}\right)^{N / 2} .
$$

Thus, if we choose $\epsilon=1$ and $C>\max \left\{A_{1}, B_{1}\right\}\left(1+\nu_{0}\right)^{N / 2}$, the condition of Theorem 2.5 also is satisfied in this case.

In the rest of this section, we consider the critical case $\beta+\delta_{2}=N$ (cf. [2; §4]). Let $(u(t), v(t)) \in E_{T}$ be a nontrivial solution of (1). By Lemma 3.1, we can assume

$$
v_{0}(x) \geq C e^{-\mu|x|^{2}}
$$

for some $C>0$ and $\mu>0$. Then by a semigroup property of $S(t)$, we have

$$
v(x, t) \geq S(t) v_{0}(x) \geq C(4 \mu t+1)^{-N / 2} e^{-|x|^{2} /(4 t+1 / \mu)} .
$$

Lemma 3.3. We have

$$
u(x, t) \geq C t^{1+\sigma_{1} / 2}(t+1)^{-N p / 2} e^{-|x|^{2} / t} .
$$

Proof. It follows from (10) and (14) that

$$
\begin{aligned}
u(x, t) & \geq \int_{0}^{t} S(t-s)|x|^{\sigma_{1}} v(x, s)^{p} d s \\
& \geq C^{p} \int_{0}^{t}(4 s+1 / \mu)^{-N p / 2} S(t-s)|x|^{\sigma_{1}} e^{-p|x|^{2} /(4 s+1 / \mu)} d s .
\end{aligned}
$$


By Lemma 3.2 with $\nu=p /(4 s+1 / \mu)$, we then have

$$
\begin{aligned}
u(x, t) & \geq C \int_{0}^{t}(4 s+1 / \mu)^{-N p / 2}(t-s)^{\sigma_{1} / 2}\left\{\frac{2 p(t-s)}{4 s+1 / \mu}+1\right\}^{-\left(N+\sigma_{1}\right) / 2} e^{-|x|^{2} / 2(t-s)} d s \\
& \geq C(2 t+1 / \mu)^{-N p / 2}(t / 2)^{\sigma_{1} / 2} e^{-|x|^{2} / t} \int_{t / 4}^{t / 2} d s
\end{aligned}
$$

Thus, the inequality of the lemma holds.

Lemma 3.4. We have

$$
v(x, t) \geq C t^{-N / 2} e^{-|x|^{2} / t} \log (t / 2 a) \quad \text { for } t \geq a
$$

where $a>0$ is a small constant.

Proof. It follows from Lemmas 3.2 and 3.3 that

$$
\begin{aligned}
v(x, t) \geq & \int_{0}^{t} S(t-s)|x|^{\sigma_{2}} u(x, s)^{q} d s \\
\geq & C \int_{0}^{t} s^{\left(1+\sigma_{1} / 2\right) q}(s+1)^{-N p q / 2}(t-s)^{\sigma_{2} / 2} \\
& \quad \times\left\{\frac{2 q(t-s)}{s}+1\right\}^{-\left(N+\sigma_{2}\right) / 2} e^{-|x|^{2} / 2(t-s)} d s \\
\geq & C(t / 2)^{\sigma_{2} / 2} t^{-\left(N+\sigma_{2}\right) / 2} e^{-|x|^{2} / t} \int_{a}^{t / 2} s^{\left\{-N(p q-1)+\left(2+\sigma_{1}\right) q+\sigma_{2}\right\} / 2} d s
\end{aligned}
$$

for small $a>0$. Since

$$
N(p q-1)-\left(2+\sigma_{1}\right) q-\sigma_{2}=(p q-1)\left(N-\beta-\delta_{2}\right)+2=-2,
$$

this proves the inequality of the lemma.

Proof of Theorem 1 (critical case). Let $(u(t), v(t)) \in E_{T}$ be a nontrivial solution of (1). Then it follows from Lemma 3.4 that

$$
[S(t) v(\cdot, t)](0) \geq C t^{-N} \log (t / 2 a) \int_{\mathbf{R}^{N}} e^{-5|x|^{2} / 4 t} d x \geq C t^{-N / 2} \log (t / 2 a)
$$

for $a<t<T$.

Contrary to the conclusion, assume that $(u(t), v(t))$ is global. Then, by Theorem 2.5,

$$
G_{\epsilon}(t)=(\epsilon / \pi)^{N / 2} \int_{\mathbf{R}^{N}} v(x, t) e^{-\epsilon|x|^{2}} d x \leq B_{1} \epsilon^{\left(\beta+\delta_{2}\right) / 2}
$$

for any $t \geq 0$ and $\epsilon>0$. Thus, choosing $\epsilon=1 / 4 t$, we obtain

$$
G_{1 / 4 t}(t)=[S(t) v(\cdot, t)](0) \leq B(4 t)^{-\left(\beta+\delta_{2}\right) / 2}=B_{1}(4 t)^{-N / 2} .
$$

This and (15) contradict each other if $T=\infty$, and the proof of Theorem 1 is complete. 


\section{Proof of Theorem 3}

First note that condition (3) can be replaced by $\left(u_{0}, v_{0}\right) \in I^{\tilde{a}} \times I^{\tilde{b}}$ since we have $I^{a} \times I^{b} \subset I^{\tilde{a}} \times I^{\tilde{b}}$. Then, to establish Theorem 3 , we have only to consider the special case $\tilde{a}=a$ and $\tilde{b}=b$.

Here we repeat condition (5):

$$
\alpha+\delta_{1}<a<\min \left\{N, N p-2-\sigma_{1}\right\}, \quad \frac{a+2+\sigma_{1}}{p}<b<a q-2-\sigma_{2} .
$$

As is easily seen, this condition is equivalent to

$$
a<N, \quad \min \{N, b\} p-a-\sigma_{1}>2, \quad a q-b-\sigma_{2}>2 .
$$

For the sake of simplicity, we set for $\gamma>0$,

$$
\eta_{\gamma}(x, t)=S(t)\langle x\rangle^{-\gamma}
$$

Lemma 4.1. We have

$$
\eta_{a}(x, t)^{-1} \leq C \max \left\{\langle x\rangle^{a},(1+t)^{a / 2}\right\} .
$$

Proof. First assume that $t<1$. As is well known, $\eta_{a}(x, t) \rightarrow\langle x\rangle^{-a}$ as $t \rightarrow 0$ locally uniformly in $x \in \mathbf{R}^{N}$. If $|x|>2$,

$$
\begin{aligned}
\eta_{a}(x, t) & \geq(4 \pi t)^{-N / 2} \int_{|y|<1} e^{-|y|^{2} / 4 t}\langle x-y\rangle^{-a} d y \\
& \geq C\langle x\rangle^{-a} \int_{|y|<1} e^{-|y|^{2} / 4} d y \geq C\langle x\rangle^{-a} .
\end{aligned}
$$

Next, let $t \geq 1$. Then we have

$$
\eta_{a}(x, t) \geq(4 \pi)^{-N / 2} t^{-a / 2} \int_{\mathbf{R}^{N}} e^{-|y|^{2} / 4}\left\langle x / t^{1 / 2}-y\right\rangle^{-a} d y .
$$

If $|x| / t^{1 / 2}<1$, this shows

$$
\eta_{a}(x, t) \geq C t^{-a / 2} \int_{\mathbf{R}^{N}} e^{-|y|^{2} / 4}\langle y\rangle^{-a} d y \geq C t^{-a / 2} .
$$

On the other hand, if $|x| / t^{1 / 2}>1$, then $\eta_{a}(x, t)>0$, and

$$
\begin{aligned}
|x|^{a} \eta_{a}(x, t) & \geq C \int_{|y|<1 / 2} e^{-|y|^{2} / 4}\left(|x| / t^{1 / 2}\right)^{a}\left\langle|x| / t^{1 / 2}-y\right\rangle^{-a} d y \\
& \rightarrow C \int_{|y|<1 / 2} e^{-|y|^{2} / 4} d y>0 \quad \text { as } \xi \rightarrow \infty
\end{aligned}
$$

Summarizing these results, we obtain the inequality in the lemma.

Lemma 4.2. We have in $\mathbf{R}^{N} \times(0, \infty)$,

$$
\begin{gathered}
|x|^{\sigma_{1}} \eta_{b}(x, t)^{p} \leq \begin{cases}C(1+t)^{\left(\sigma_{1}+a-\min \{N, b\} p\right) / 2} \eta_{a}(x, t), & \text { if } b \neq N \\
C(1+t)^{\left(\sigma_{1}+a-N p\right) / 2}[\log (2+t)]^{p} \eta_{a}(x, t), & \text { if } b=N\end{cases} \\
|x|^{\sigma_{2}} \eta_{a}(x, t)^{q} \leq C(1+t)^{\left(\sigma_{2}+b-a q\right) / 2} \eta_{b}(x, t) .
\end{gathered}
$$


Proof. We only consider the case $b \geq a$. A similar argument can be applied also to the case $b \leq a$. We have by Lemma 4.1:

$$
\begin{aligned}
|x|^{\sigma_{1}} \eta_{b}(x, t)^{p} & =|x|^{\sigma_{1}} \eta_{b}(x, t)^{p} \eta_{a}(x, t)^{-1} \eta_{a}(x, t) \\
& \leq C|x|^{\sigma_{1}} \max \left\{\langle x\rangle^{a},(1+t)^{a / 2}\right\} \eta_{b}(x, t)^{p} \eta_{a}(x, t) .
\end{aligned}
$$

Since $\left(a+\sigma_{1}\right) / p \leq \min \{N, b\}$ by (17), we can use Lemma 2.1 to obtain (18).

Next, by use of the Jensen inequality, we have

$$
|x|^{\sigma_{2}} \eta_{a}(x, t)^{q} \leq|x|^{\sigma_{2}} \eta_{a}(x, t)^{q-r} \eta_{b}(x, t)
$$

where $r=b / a$. Since $\sigma_{2} \leq a(q-r)=a q-b$ by (17), Lemmas 4.1 and 2.1 also give (19).

We define the Banach space $E_{\eta}$ of pairs $(u(t), v(t))$ such that

$$
\|(u, v)\|_{E_{\eta}} \equiv\left\|u / \eta_{a}\right\|_{\infty}+\left\|v / \eta_{b}\right\|_{\infty}<\infty
$$

where

$$
\left\|w\left|\|_{\infty}=\sup _{(x, t) \in \mathbf{R}^{N} \times(0, \infty)}\right| w(x, t) \mid\right.
$$

and consider again the integral equation (10) in $E_{\eta}$.

Lemma 4.3. (i) Let $\left(u_{0}, v_{0}\right)$ satisfy (3). Then we have $\left(S(\cdot) u_{0}, S(\cdot) v_{0}\right) \in E_{\eta}$ and

$$
\left\|\left(S(\cdot) u_{0}, S(\cdot) v_{0}\right)\right\|_{E_{\eta}} \leq\left\{\left\|u_{0}\right\|_{\infty, a}+\left\|v_{0}\right\|_{\infty, b}\right\} .
$$

(ii) Let $(u, v) \in E_{\eta}$. Then we have $\left(\Phi_{1}(v), \Phi_{2}(u)\right) \in E_{\eta}$ and

$$
\left\|\left(\Phi_{1}(v), \Phi_{2}(u)\right)\right\|_{E_{\eta}} \leq C\left\{\|(0, v)\|_{E_{\eta}}^{p}+\|(u, 0)\|_{E_{\eta}}^{q}\right\} .
$$

Proof. (i) is obvious from the definition of $E_{\eta}$.

(ii) We have from (18):

$$
\begin{aligned}
\left|\Phi_{1}(v)\right| & \leq \int_{0}^{t} S(t-s)|\cdot|^{\sigma_{1}}|v(s)|^{p} d s \\
& \leq \int_{0}^{t} S(t-s)|x|^{\sigma_{1}} \eta_{b}(x, s)^{p} d s\left\|v / \eta_{b}\right\|_{\infty}^{p} \\
& \leq C \eta_{a}(x, t)\left\|v / \eta_{b}\right\|_{\infty}^{p}\left\{\begin{array}{l}
\int_{0}^{t}(1+s)^{\left(\sigma_{1}+a-\min \{N, b\} p\right) / 2} d s \quad \text { or } \\
\int_{0}^{t}(1+s)^{\left(\sigma_{1}+a-N p\right) / 2} \log (2+s)^{p} d s,
\end{array}\right.
\end{aligned}
$$

and from (19):

$$
\left|\Phi_{2}(u)\right| \leq C \eta_{b}(x, t) \int_{0}^{t}(1+s)^{\left(\sigma_{2}+b-a q\right) / 2} d s\left\|u / \eta_{a}\right\|_{\infty}^{q} .
$$

Thus, noting (17), we obtain

$$
\left|\Phi_{1}(v)\right| \leq C \eta_{a}(x, t)\left\|v / \eta_{b}\right\|_{\infty}^{p}, \quad\left|\Phi_{2}(u)\right| \leq C \eta_{b}(x, t)\left\|\mid u / \eta_{a}\right\|_{\infty}^{q},
$$

which imply the desired conclusion.

Proof of Theorem 3 (the case $p>1$ ). We consider the map $\Psi$ defined by (11) in $E_{\eta}$. Let $\left\|u_{0}\right\|_{\infty, a}+\left\|v_{0}\right\|_{\infty, b}=m>0$ and set $B_{m}=\left\{(u, v) \in E_{\eta} \mid\|(u, v)\|_{E_{\eta}} \leq 2 m\right\}$ and $P_{\eta}=\left\{(u, v) \in E_{\eta} \mid u \geq 0, v \geq 0\right\}$. Then since $p, q>1$, by means of the above lemma, we easily see that $\Psi$ is a strict contraction of $B_{m} \cap P_{\eta}$ into itself provided $m$ is small enough, whence the result. 
Next, we consider the case $p=1$. In this case, (10) is reduced to

$$
u(t)=T(t)\left(u_{0}, v_{0}\right)+\Gamma(u)
$$

where

$$
\begin{gathered}
T(t)\left(u_{0}, v_{0}\right)=S(t) u_{0}+\int_{0}^{t} S(t-s)|\cdot|^{\sigma_{1}} S(s) v_{0} d s \\
\Gamma(u)=\int_{0}^{t} S(t-s)|\cdot|^{\sigma_{1}} \int_{0}^{s} S(s-\tau)|t|^{\sigma_{2}}|u(\tau)|^{q-1} u(\tau) d \tau d s .
\end{gathered}
$$

We shall consider equation (20) in the Banach space $X$ with norm $\left\|u / \eta_{a}\right\|_{\infty}$.

Lemma 4.4. (i) Let $\left(u_{0}, v_{0}\right)$ satisfy (3). Then $T(\cdot)\left(u_{0}, v_{0}\right) \in X$ and

$$
\left\|T(\cdot)\left(u_{0}, v_{0}\right) / \eta_{a}\right\|_{\infty} \leq C\left\{\left\|u_{0}\right\|_{\infty, a}+\left\|v_{0}\right\|_{\infty, b}\right\} .
$$

(ii) $\Gamma$ maps $X$ into itself and

$$
\|\Gamma(u)\|_{\infty} \leq C\|u\|_{\infty}^{q} \quad \text { for } u \in X .
$$

Proof. (i) As in the proof of Lemma 4.2 (ii), noting (17), we have from (18):

$$
\begin{aligned}
\left|T(t)\left(u_{0}, v_{0}\right)\right| & \leq \eta_{a}(x, t)\left\|u_{0}\right\|_{\infty, a}+\int_{0}^{t} S(t-s)|\cdot|{ }^{\sigma_{1}} \eta_{b}(\cdot, s) d s\left\|v_{0}\right\|_{\infty, b} \\
& \leq \eta_{a}(x, t)\left\{\left\|u_{0}\right\|_{\infty, a}+C\left\|v_{0}\right\|_{\infty, b}\right\} .
\end{aligned}
$$

This implies assertion (i).

(ii) Similarly as above, it follows from (17) and (19) that

$$
|\Gamma(u)| \leq C \int_{0}^{t} S(t-s)|\cdot|^{\sigma_{1}} \eta_{b}(s) d s\left\|u / \eta_{a}\right\|_{\infty}^{q} .
$$

Moreover, it follows from (17) and (18) that

$$
|\Gamma(u)| \leq C \eta_{a}(x, t)\left\|u / \eta_{a}\right\|_{\infty}^{q} .
$$

Thus, assertion (ii) is concluded.

Proof of Theorem 3 (the case $p=1$ ). We consider the map $v \rightarrow T(\cdot)\left(u_{0}, v_{0}\right)+\Gamma(v)$ in $X$. Let $\left\|u_{0}\right\|_{\infty, a}+C\left\|v_{0}\right\|_{\infty, b}=m>0$ and set $B_{m}=\left\{u \in X \mid\left\|u / \eta_{a}\right\|_{\infty} \leq 2 m\right\}$ and $P=\{u \in X \mid u \geq 0\}$. Then since $q>1$, by means of the above lemma, we easily see that this map is a strict contraction of $B_{m} \cap P$ into itself provided $m$ is small enough. Hence, there exists a unique fixed point $u \in X$ which solves (20). We substitute this $u$ in the second equality of $(10)$. Then the pair $(u, v)$ solves $(10)$. Note that by Lemma 4.3 ,

$$
|v(t)| \leq \eta_{b}(x, t)\left\{\left\|v_{0}\right\|_{\infty, b}+C \mid\left\|u / \eta_{a}\right\|_{\infty}^{q}\right\} .
$$

Thus, we conclude $(u, v) \in E_{\eta}$, and the proof of Theorem 3 is complete. 


\section{Proof of Theorem 4}

In this section, we follow the argument of Kamin and Peletier [10], who studied the asymptotic behavior of the heat equation with nonlinear absorption.

We put

$$
u_{k}(x, t)=k^{a} u\left(k x, k^{2} t\right), \quad v_{k}(x, t)=k^{b^{\prime}} v\left(k x, k^{2} t\right)
$$

for $k>0$ where $b^{\prime}=\min \{N, b\}$. Then $\left(u_{k}(t), v_{k}(t)\right)$ solves

$$
\begin{gathered}
u_{k t}=\Delta u_{k}+k^{a+2+\sigma_{1}-b^{\prime} p}|x|^{\sigma_{1}} v_{k}^{p}, \\
v_{k t}=\Delta v_{k}+k^{b^{\prime}+2+\sigma_{2}-a q} u_{k}^{q}, \\
u_{k}(x, 0)=k^{a} u_{0}(k x), \quad v_{k}(x, 0)=k^{b^{\prime}} v_{0}(k x) .
\end{gathered}
$$

Note that we have assumed $a<N$ and $b \neq N$. Then it follows from (4) that

$$
\begin{gathered}
\left\|u_{k}(t)\right\|_{\infty} \leq k^{a} C\left(k^{2} t\right)^{-a / 2}=C t^{-a / 2}, \\
\left\|v_{k}(t)\right\|_{\infty} \leq k^{b^{\prime}} C\left(k^{2} t\right)^{-b^{\prime} / 2}=C t^{-b^{\prime} / 2} .
\end{gathered}
$$

Thus, $\left\{u_{k}(x, t)\right\}$ and $\left\{v_{k}(x, t)\right\}$ are uniformly bounded in $\mathbf{R}^{N} \times[\delta, \infty)$ for any $\delta>0$. As is easily seen from the integral equation (10), the uniform boundedness implies the equicontinuity of $\left\{u_{k}(x, t)\right\}$ and $\left\{v_{k}(x, t)\right\}$ in any bounded set of $\mathbf{R}^{N} \times[\delta, \infty)$. Then, using the Ascoli-Arzela theorem and a diagonal sequence method in $\delta$, we see that for any sequence $\left\{k_{j}\right\} \rightarrow \infty$, there exists a subsequence $\left\{k_{j}^{\prime}\right\}$ and continuous functions $w_{1}(x, t), w_{2}(x, t)$ such that

$$
u_{k_{j}^{\prime}}(x, t) \rightarrow w_{1}(x, t), \quad v_{k_{j}^{\prime}}(x, t) \rightarrow w_{2}(x, t) \quad \text { as } k_{j}^{\prime} \rightarrow \infty
$$

locally uniformly in $\mathbf{R}^{N} \times(0, \infty)$.

Proof of Theorem 4. (i) We shall first show

$$
w_{1}(x, t)=S(t)|x|^{-a} \text {. }
$$

It follows from the first equation of (21) that

$$
\begin{aligned}
\int_{\mathbf{R}^{N}} & u_{k}(x, t) \zeta(x, t) d x-\int_{\mathbf{R}^{N}} u_{k}(x, 0) \zeta(x, 0) d x \\
& =\int_{0}^{t} \int_{\mathbf{R}^{N}}\left\{u_{k} \zeta_{t}+u_{k} \Delta \zeta+k^{a+2+\sigma_{1}-b^{\prime} p}|\cdot|^{\sigma_{1}} v_{k}^{p} \zeta\right\} d x d t
\end{aligned}
$$

for any $t>0$ and nonnegative test function $\zeta(x, t) \in C_{0}^{\infty}\left(\mathbf{R}^{N} \times[0, \infty)\right)$. By assumption (6) on the initial value $u_{0}$,

$$
\begin{aligned}
\int_{\mathbf{R}^{N}} u_{k}(x, 0) \zeta(x, 0) d x & =\int_{\mathbf{R}^{N}} k^{a} u_{0}(k x) \zeta(x, 0) d x \\
& \rightarrow \int_{\mathbf{R}^{N}} A|x|^{-a} \zeta(x, 0) d x \text { as } k=k_{j}^{\prime} \rightarrow \infty .
\end{aligned}
$$

On the other hand,

$$
\begin{aligned}
\int_{0}^{t} \int_{\mathbf{R}^{N}} k^{a+2+\sigma_{1}-b^{\prime} p}|\cdot|^{\sigma_{1}} v_{k}^{p} \zeta d x d t \\
\quad=\int_{0}^{k^{2} t} \int_{\mathbf{R}^{N}} k^{a+\sigma_{1}}|x|^{\sigma_{1}} v(k x, \tau)^{p} \zeta\left(x, k^{-2} \tau\right) d x d \tau .
\end{aligned}
$$


Here,

$$
\begin{aligned}
k^{a+\sigma_{1}}|x|^{\sigma_{1}} v(k x, \tau)^{p}= & {\left[(k|x|)^{b^{\prime}} v(k x, \tau)\right]^{\left(a+\sigma_{1}\right) \rho / b^{\prime}} } \\
& \times v^{p-\left(a+\sigma_{1}\right) \rho / b^{\prime}} k^{\left(a+\sigma_{1}\right)(1-\rho)}|x|^{\sigma_{1}-\left(a+\sigma_{1}\right) \rho}
\end{aligned}
$$

for $\rho>1$. As is easily seen from (7) and Lemma 2.1, $(k|x|)^{b^{\prime}} v(k x, \tau)$ is bounded in $\mathbf{R}^{N} \times(0, \infty)$ and

$$
v(k x, \tau)^{p-\left(a+\sigma_{1}\right) \rho / b^{\prime}} \leq C(1+t)^{\left(-b^{\prime} p+a+\sigma_{1}\right) / 2} .
$$

By assumption (17), there exists a $\rho>1$ such that

$$
a \rho+\sigma_{1}(\rho-1)<N \text { and } b^{\prime} p-\left(a+\sigma_{1}\right) \rho>2 .
$$

Then, since $\left(a+\sigma_{1}\right)(1-\rho)<0$, these imply

$$
\int_{0}^{k^{2} t} \int_{\mathbf{R}^{N}} k^{a+\sigma_{1}}|x|^{\sigma_{1}} v(k x, \tau)^{p} \zeta\left(x, k^{-2} \tau\right) d x d \tau \rightarrow 0 \quad \text { as } \quad k=k_{j}^{\prime} \rightarrow \infty .
$$

Thus, letting $k=k_{j}^{\prime} \rightarrow \infty$ in (23), we obtain

$$
\int_{\mathbf{R}^{N}} w_{1}(x, t) \zeta(x, t) d x-\int_{\mathbf{R}^{N}} A|x|^{-a} \zeta(x, 0) d x=\int_{0}^{t} \int_{\mathbf{R}^{N}}\left\{w_{1} \zeta_{t}+w_{1} \Delta \zeta\right\} d x d t .
$$

The uniqueness of solutions of

$$
u_{t}=\Delta u, \quad u(x, 0)=A|x|^{-a},
$$

then gives (22).

The uniqueness result asserts more:

$$
u_{k}(x, t) \rightarrow A S(t)|x|^{-a} \quad \text { as } \quad k \rightarrow \infty
$$

uniformly in compact sets of $\mathbf{R}^{N} \times(0, \infty)$.

Note again (4), that is,

$$
u_{k}(x, t) \leq C k^{a} S\left(k^{2} t\right)|k x|^{-a} .
$$

Let $t=1$ in this inequality. Then, by the self-similarity of $S(t)|x|^{-a}$, we have

$$
u_{k}(x, 1) \leq C S(1)|x|^{-a} \text {. }
$$

This inequality implies that for any $\epsilon>0$, there exists an $R>0$ independent of $k>1$ such that $\left\{u_{k}(x, 1)\right\}$ are uniformly less than $\epsilon$ in $|x|>R$. Therefore, it follows from (24) that

$$
u_{k}(x, 1)-A S(1)|x|^{a} \rightarrow 0 \quad \text { as } \quad k \rightarrow \infty
$$

uniformly in $\mathbf{R}^{N}$. We let $y=k x$ and $s=k^{2}$ in this relation. Then noting again the self-similarity of $S(t)|x|^{-a}$, we conclude that

$$
\left.s^{a / 2}|u(y, s)-A S(s)| y\right|^{-a} \mid \rightarrow 0 \quad \text { as } \quad s \rightarrow \infty
$$

uniformly in $\mathbf{R}^{N}$.

Relation (7) now is proved for $u(x, t)$. The same argument can be applied also to $v(x, t)$ if $b<N$ and (6) is satisfied by $v_{0}(x)$.

Proof of Theorem 4 (i) is now complete. 
Proof of Theorem 4. (ii) As in the above case, we shall show

$$
u_{k}(x, t) \rightarrow w_{2}(x, t)=M(4 \pi t) e^{-|x|^{2} / 4 t} \quad \text { as } \quad k \rightarrow \infty
$$

locally uniformly in $\mathbf{R}^{N} \times(0, \infty)$ where $M$ is as given in (11). It follows from the second equation of $(20)$ that

$$
\begin{aligned}
\int_{\mathbf{R}^{N}} & v_{k}(x, t) \zeta(x, t) d x-\int_{\mathbf{R}^{N}} v_{k}(x, 0) \zeta(x, 0) d x \\
& =\int_{0}^{t} \int_{\mathbf{R}^{N}}\left\{v_{k} \zeta_{t}+v_{k} \Delta \zeta+k^{N+2+\sigma_{2}-a q}|\cdot|^{\sigma_{2}} u_{k}^{q} \zeta\right\} d x d t
\end{aligned}
$$

for any $t>0$ and nonnegative $\zeta(x, t) \in C_{0}^{\infty}\left(\mathbf{R}^{N} \times[0, \infty)\right)$.

Since $b>N$, condition (3) implies that $v_{0}(x) \in L^{1}$. Then we have

$$
\int_{\mathbf{R}^{N}} v_{k}(x, 0) \zeta(x, 0) d x=\int_{\mathbf{R}^{N}} v_{0}(x) \zeta\left(k^{-1} x, 0\right) d x \rightarrow \int_{\mathbf{R}^{N}} v_{0}(x) d x \zeta(0,0)
$$

as $k=k_{j}^{\prime} \rightarrow \infty$. On the other hand,

$$
\int_{0}^{t} \int_{\mathbf{R}^{N}} k^{N+2+\sigma_{2}-a q}|\cdot|^{\sigma_{2}} u_{k}^{q} \zeta d x d t=\int_{0}^{k^{2} t} \int_{\mathbf{R}^{N}}|x|^{\sigma_{2}} u(x, \tau)^{q} \zeta\left(k^{-1} x, k^{-2} \tau\right) d x d \tau .
$$

Here (4) and Lemma 2.1 lead to

$$
|x|^{\sigma_{2}} u(x, \tau)^{q} \leq C(1+\tau)^{\left(-a(q-r)+\sigma_{2}\right) / 2} u(x, \tau)^{r}
$$

for some $r$ satisfying $\sigma_{2} \leq a(q-r)$. We put $r=N \rho / a$. Then, by assumption (17), we can choose $\rho>1$ to satisfy

$$
a(q-r)-\sigma_{2}=a q-N \rho-\sigma_{2}>2 .
$$

Then, since

$$
u(x, \tau)^{r} \leq C\left[S(t)\langle x\rangle^{-a}\right]^{r} \leq C S(t)\langle x\rangle^{-N \rho}
$$

it follows that

$$
\int_{0}^{\infty} \int_{\mathbf{R}^{N}}|x|^{\sigma_{2}} u(x, \tau)^{q} d x d \tau<\infty
$$

and we have

$$
\int_{0}^{k^{2} t} \int_{\mathbf{R}^{N}}|x|^{\sigma_{2}} u(x, \tau)^{q} \zeta\left(k^{-1} x, k^{-2} \tau\right) d x d \tau \rightarrow \int_{0}^{\infty} \int_{\mathbf{R}^{N}}|x|^{\sigma_{2}} u(x, \tau)^{q} d x d \tau \zeta(0,0)<\infty
$$

as $k=k_{j}^{\prime} \rightarrow \infty$. Thus, letting $k=k_{j}^{\prime} \rightarrow \infty$ in (26), we obtain

$$
\int_{\mathbf{R}^{N}} w_{2}(x, t) \zeta(x, t) d x-M \zeta(0,0)=\int_{0}^{t} \int_{\mathbf{R}^{N}}\left\{w_{2} \zeta_{t}+w_{2} \Delta \zeta\right\} d x d t
$$

The uniqueness of solutions of

$$
u_{t}=\Delta u, \quad u(x, 0)=M \delta(x)
$$

where $\delta(x)$ is the Dirac $\delta$-function, then implies (25).

We put $t=1$ in (25). Then letting $y=k x$ and $s=k^{2}$, we conclude

$$
s^{a / 2}\left|u(y, s)-M(4 \pi s)^{-N / 2} e^{-|y|^{2} / 4 s}\right| \rightarrow 0 \quad \text { as } \quad s \rightarrow \infty
$$

uniformly in $\left\{y \in \mathbf{R}^{N}|| y \mid \leq R s^{1 / 2}\right\}$ for any $R>0$.

Theorem 4 (ii) thus is proved. 
Acknowledgments. We would like to thank the referees for their careful reading of the manuscript and their suggestions.

\section{References}

1. C. Bandle and H. A. Levine, On the existence and nonexistence of global solutions of reactiondiffusion equations in sectorial domains, Trans. Amer. Math. Soc. 316 (1989), 595-622.

2. M. Escobedo and M. A. Herrero, Boundedness and blow up for a semilinear reaction-diffusion system, J. Diff. Eqns. 89 (1991), 176-202.

3. H. Fujita, On the blowing up solutions of the Cauchy problem for $u_{t}=\delta u+u^{1+\alpha}$, J. Fac. Sci. Univ. Tokyo 13 (1966), 109-124.

4. V. A. Galktionov, S. P. Kurdyumov, and A. A. Samarskii, A parabolic system of quasilinear equations I, Differential Equations 19 (1983), 2133-2143.

5. __ A parabolic system of quasilinear equations II, Differential Equations 21 (1985), 15441559.

6. T. Hamada, Nonexistence of global solutions of parabolic equations in conical domains, Tsukuba J. Math., 21 (1997), 505-514.

7. K. Hayakawa, On nonexistence of global solutions of some semilinear parabolic equations, Proc. Japan Acad. 49 (1973), 503-505.

8. Q. Huang, K. Mochizuki, and K. Mukai, Life span and asymptotic behavior for a semilinear parabolic system with slow decay initial data, Hokkaido Math. J., to appear.

9. T. Imai and K. Mochizuki, On blow-up of solutions for quasilinear degenerate parabolic equations, Publ. RIMS, Kyoto Univ. 27 (1991), 695-709.

10. S. Kamin and L. A. Peletier, Large time behaviour of solutions of the heat equation with absorption, Ann. Scu. Norm. Sup. Pisa XII (1985), 393-408.

11. K. Kobayashi, T. Sirao, and H. Tanaka, On blowing up problem for semilinear heat equations, J. Math. Soc. Japan 29 (1977), 407-424.

12. T.-Y. Lee and W.-M. Ni, Global existence, large time behavior, and life span on solutions of a semilinear parabolic Cauchy problem, Trans. Amer. Math. Soc. 333 (1992), 365-378.

13. K. Mochizuki, Global existence, nonexistence, and asymptotic behavior for quasilinear parabolic equations, Proc. Sixth Tokyo Conf. on Nonlinear PDE (H. Ishii Ed.), 1997, 22-27.

14. Y.-W. Qi and H. A. Levine, The critical exponent of degenerate parabolic systems, Z. angew Math. Phys. 44 (1993), 249-265.

15. Y. Uda, The critical exponent for a weakly coupled system of the generalized Fujita-type reactiondiffusion equations, preprint 1996.

16. F. B. Weissler, Existence and nonexistence of global solutions for semilinear heat equation, Israel J. Math. 38 (1981), 29-40.

Department of Mathematics, Tokyo Metropolitan University, Hachioji, Tokyo 192-03, JAPAN 